Renate NITSCH, Darmstadt

\title{
Diagnose von Lernschwierigkeiten im Bereich funktionaler Zusammenhänge
}

Im Umgang mit funktionalen Zusammenhängen zeigen Schülerinnen und Schüler vielfältige Verständnisschwierigkeiten. Diese lassen sich meist auf konzeptuelle Schwierigkeiten mit Begriffen, Zusammenhängen und Verfahren zurückführen und sind durch die Analyse systematischer Fehler aufdeckbar. Systematische Fehler zeichnen sich dadurch aus, dass sie im Gegensatz zu Flüchtigkeitsfehlern, die zufällig auftreten und meist aufgrund von Konzentrationsmangel entstehen, reproduzierbar sind und auf fehlerhaften Vorstellungen und Konzepten beruhen (Radatz, 1980). Diese werden im Bereich der Naturwissenschaften auf individuelle Alltagsvorstellungen der Schülerinnen und Schüler zurückgeführt, in der Mathematik sind jedoch auch innermathematische Fehlvorstellungen denkbar. Eine weitere Besonderheit zeigt sich bei der Untersuchung typischer Fehlermuster. Entgegen bisheriger Annahmen sind diese häufig nicht konsistent, was vermuten lässt, dass Lösungswege teilweise ad hoc gebildet werden (Wittmann, 2012). Dies wirft die Frage auf, wie sich Lernschwierigkeiten im Zusammenhang mit den beobachteten Phänomenen lerntheoretisch erfassen lassen.

\section{Lerntheoretisches Konzept zur Entstehung von Lernschwierigkeiten}

Das Tätigkeitskonzept nach Vygotskij und Leont'ev stellt ein geeignetes lerntheoretisches Modell zur Beschreibung von Lernhandlungen bei der Aneignung individueller Kenntnisse und Vorstellungen zur Verfügung. Es werden explizit Mechanismen zur Orientierungsbildung bezüglich einer gestellten Anforderung analysiert. Eine (Lern-)Handlung wird demnach unterteilt in einen orientierenden und einen ausführenden Teil, wobei sich der orientierende Teil auf eine bestimmte Orientierungsgrundlage stützt (Galperin, 1973). Diese entsteht immer unmittelbar in Bezug auf eine aktuelle Anforderung und greift auf vorhandene Kenntnisse und Vorstellungen zurück. Sie kann auf drei Orientierungsleveln ausgebildet werden, hier im Vokabular nach Bruder: Auf der Ebene der Probierorientierung sind einzelne Kenntnisse und Vorstellungen abrufbar, aber sie stehen in keiner geordneten Struktur. Das Vorgehen ist unbeständig und gekennzeichnet durch ein Lernen nach Versuch und Irrtum. Dabei kann die Aufgabenbearbeitung auch zum Erfolg führen, allerdings ist sie meist mit Fehlern, Umwegen oder Wiederholungen verbunden. Auf der Ebene der Musterorientierung sind vertraute Handlungsmuster im Rahmen einer empirischen Orientierung verfügbar. Die Vorgehensweise ist durch das Abarbeiten bekannter 
Schemata bestimmt, die beispielsweise durch bereits gelöste Beispielaufgaben desselben Typs als Muster gespeichert sind. Der Lernende erfasst alle wesentlichen Merkmale und Bedingungen der Handlung, allerdings immer in Bezug auf eine konkrete Anforderung. Aufgrund des geringen Verallgemeinerungsgrades ist eine Übertragung der Handlung beschränkt auf analoge Aufgaben. Die Ebene der Feldorientierung zeichnet sich durch eine verallgemeinerte Orientierung aus. Der Lernende kann die erforderlichen Schritte und Bedingungen der Handlung selbst ableiten. Folglich sind größere Transferleistungen möglich, indem Kenntnisse und Vorstellungen auch auf weniger vertraute Anforderungssituationen übertragen werden.

Lernschwierigkeiten lassen sich innerhalb dieses Konzeptes mit einer unvollständigen Orientierungsgrundlage, d.h. auf Ebene der Probier- und Musterorientierung erklären. Scheitert ein Schüler bei seinem Lösungsversuch einer konkreten Anforderung, so kann dies zum einen darin begründet sein, dass er sich auf Ebene der Probierorientierung befindet. Er hat demnach weder Handlungsmuster in Bezug auf die aktuelle Anforderung verfügbar, noch besitzt er die notwendigen Fähigkeiten zur Handlungsbewältigung. Auf der Ebene der Musterorientierung können Lernschwierigkeiten mit der Aktivierung inadäquater Muster erklärt werden. Ist die Passung zwischen Anforderung und aktiviertem Muster nicht korrekt, kann es zu einer fehlerhaften Lösung kommen. Dabei kann der Schüler durchaus über ein passendes Handlungsmuster verfügen, bezüglich der aktuellen Anforderung greift er jedoch auf ein anderes Muster zurück. Dies lässt vermuten, dass das zutreffende Muster noch nicht ausreichend verfestigt ist und bei einer leichten Abänderung der Anforderung womöglich frühere Muster dominieren. Im Bereich funktionaler Zusammenhänge ist dies zum Beispiel zu beobachten, wenn zur Bearbeitung von Problemstellungen zu quadratischen Funktionen lineare Funktionen herangezogen werden. Um eine Fehlvorstellung handelt es sich dann, wenn zu einem Aufgabentyp immer das gleiche fehlerhafte Muster aktiviert wird, woraus geschlossen werden kann, dass der Schüler fehlerhafte Kenntnisse bzw. Vorstellungen verinnerlicht hat. Dabei können Fehlvorstellungen sowohl als inadäquate wissenschaftliche Vorstellung als auch als inadäquate Alltagsvorstellung auftreten.

Für die Diagnose von Lernschwierigkeiten ist diese grundlegende Unterscheidung zwischen fehlenden bzw. noch nicht gefestigten und fehlerhaften Mustern wesentlich, da sich daraus unterschiedliche Interventionsmaßnahmen ergeben. Während die beiden ersten Möglichkeiten Anlass geben, mithilfe elementarer Identifizierungs- und Realisierungsaufgaben systematisch neue Vorstellungen und Kenntnisse über den mathematischen Inhalt und/oder über mögliche Handlungsmuster aufzubauen, legt die Diagnose 
einer möglichen Fehlvorstellung eine Umstrukturierung vorhandener kognitiver Strukturen nahe. Hierbei kann auf Ergebnisse der aktuellen Conceptual Change Forschung zurückgegriffen werden (Vosniadou, 2008).

\section{Bedeutung für den Inhaltsbereich funktionaler Zusammenhänge}

Bezüglich der Kenntnisse und Vorstellungen im Bereich funktionaler Zusammenhänge können nach Vollrath (1989) drei Aspekte mathematischer Funktionen besonders hervortreten: Zuordnungsaspekt, Kovariationsaspekt und Objektaspekt. Diese drei Aspekte von Funktionen bilden sich unterschiedlich stark in den verschiedenen Darstellungswechseln zwischen den Darstellungsformen algebraischer Gleichung (A), Graph (G), numerischer Wertetabelle (N) und situativer Beschreibung (S) ab. Betrachtet man die einzelnen Darstellungswechsel hinsichtlich der notwendigen Lösungsschritte, so kann man jedem einzelnen einen dominierenden Aspekt zuordnen, wobei die Richtung des Darstellungswechsels (Start- vs. Zielrepräsentation) berücksichtigt werden muss.

\begin{tabular}{|l|lll|lll|ll|}
\hline Aspekte von Funktionen & \multicolumn{2}{|c|}{ Zuordnung } & \multicolumn{2}{c|}{ Kovariation } & \multicolumn{2}{c|}{ Objekt } \\
\hline Darstellungswechsel XY & SN & NG & AN & AG & GA & NA & GS & SA \\
X=Startrepräsentation & NS & GN & & & & & SG & AS \\
Y= Zielrepräsentation & & & & & & & & \\
\hline
\end{tabular}

Beispielsweise dominiert beim Darstellungswechsel von einer Wertetabelle zu einem Graphen in beiden Richtungen (NG und GN) der Zuordnungsaspekt, da einzelne Wertepaare abgelesen und in die Wertetabelle eingetragen werden müssen. Ähnliches gilt für den Darstellungswechsel zwischen Gleichung und Wertetabelle (AN). In der umgekehrten Richtung (NA) steht die Änderung der Werte der einzelnen Koordinaten im Vordergrund, wodurch der Kovariationsaspekt betont wird. Sofern eine situative Beschreibung enthalten ist, ist häufig die Betrachtung der Funktion als Ganzes wesentlich (Objektaspekt). Durch die Zuordnung zu den Aspekten ist es möglich, über den Umgang mit verschiedenen Darstellungswechseln Rückschlüsse auf vorhandene Kenntnisse und Vorstellungen zu ziehen.

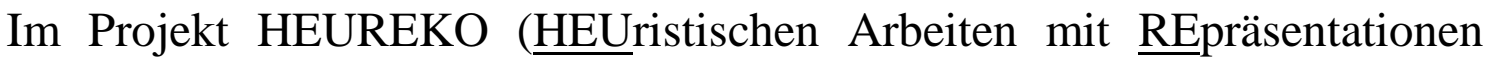
funktionaler Zusammenhänge und der Diagnose mathematischer KOmpetenzen von Schülerinnen und Schülern), in welchem ein 5-dimensionales Kompetenzstrukturmodell zu Darstellungswechseln im Bereich funktionaler Zusammenhänge empirisch bestätigt wurde (Nitsch et al., eingereicht), haben sich bereits erste Anhaltspunkte für typische Lernschwierigkeiten mit bestimmten Darstellungswechseln ergeben. Diese werden durch eine aktuelle Studie von Bossé, Adu-Gyamfi und Cheetham (2011) bestätigt. 
Demnach scheint der Zuordnungsaspekt am wenigsten Schwierigkeiten zu bereiten. Darstellungswechsel, die den Kovariationsaspekt fordern, sind für die Lernenden hingegen deutlich schwieriger zu bewältigen. Die größten Schwierigkeiten beinhalten Darstellungswechsel mit situativer Beschreibung (Objektaspekt).

\section{Ausblick auf das zu entwickelnde Diagnoseinstrument}

Auf Basis der vorangehenden Überlegungen soll im Rahmen des Projekts CODI (COnceptual DIfficulties in the field of functional relationships) ein Diagnoseinstrument zum Aufdecken typischer Lernschwierigkeiten entwickelt und empirisch erprobt werden. Hierzu werden unter Berücksichtigung der in der Literatur berichteten Schülerschwierigkeiten im Bereich funktionaler Zusammenhänge und unter Nutzung der Erkenntnisse aus dem Projekt HEUREKO Items zu verschiedenen Darstellungswechseln generiert, die auf typische Lernschwierigkeiten abzielen. Durch die Analyse konsistenter Fehlermuster sollen dabei insbesondere mögliche Fehlvorstellungen untersucht werden. Langfristig sollen die Mathematiklehrkräfte mithilfe eines online-Diagnosetools bei der Diagnose von Lernschwierigkeiten unterstützt werden, um anschließend eine individuelle Förderung zur Behebung identifizierter Lernschwierigkeiten einleiten zu können.

\section{Literatur}

Bossé, M., Adu-Gyamfi, K. \& Cheetham, M. (2011). Assessing the Difficulty of Mathematical Translations: Synthesizing the Literature and Novel Findings. International Electronic Journal of Mathematics Education, 6 (3), 113-133.

Galperin, P.J. (1973). Die Psychologie des Denkens und die Lehre von der etappenweise Ausbildung geistiger Operationen. In: Budilowa E.A. et al.: Die Psychologie des Denkens und die Lehre von der etappenweisen Ausbildung geistiger Operationen. Berlin: deb literaturvertrieb, 81-119.

Nitsch, R., Fredebohm, A., Bruder, R., Kelava, T., Naccarella, D., Leuders, T. \& Wirtz, M. (eingereicht). Students' Competencies in working with Functions in Secondary Mathematics Education - Empirical Examination of a Competence Structure Model. International Journal of Science and Mathematics Education.

Radatz, H. (1980). Fehleranalysen im Mathematikunterricht. Braunschweig: Vieweg.

Vollrath, H.J. (1989). Funktionales Denken. Journal für Mathematikdidaktik 10, 3-37.

Vosniadou, S. (2008). International Handbook of Research on Conceptual Change. New York: Routledge.

Wittmann, G. (2012). Zur Konsistenz von Fehlermustern in der Bruchrechnung - Ergebnisse einer empirischen Studie. In: Beiträge zum Mathematikunterricht 2012, Band 2. Münster: Waxmann, 945-948. 